\title{
MOMENT CONVERGENCE RATES OF LIL FOR NEGATIVELY ASSOCIATED SEQUENCES
}

\author{
Ke-Ang Fu And Li-Hua Hu
}

ABSTRACT. Let $\left\{X_{n} ; n \geq 1\right\}$ be a strictly stationary sequence of negatively associated random variables with mean zero and finite variance. Set $S_{n}=\sum_{k=1}^{n} X_{k}, M_{n}=\max _{k<n}\left|S_{k}\right|, n \geq 1$. Suppose $\sigma^{2}=\mathrm{E} X_{1}^{2}+$ $2 \sum_{k=2}^{\infty} \mathrm{E} X_{1} X_{k}(0<\sigma<\infty)$. We prove that for any $b>-1 / 2$, if $\mathrm{E}|X|^{2+\delta}(0<\delta \leq 1)$, then

$$
\begin{aligned}
& \lim _{\varepsilon \searrow 0} \varepsilon^{2 b+1} \sum_{n=1}^{\infty} \frac{(\log \log n)^{b-1 / 2}}{n^{3 / 2} \log n} \mathrm{E}\left\{M_{n}-\sigma \varepsilon \sqrt{2 n \log \log n}\right\}_{+} \\
= & \frac{2^{-1 / 2-b} \sigma \mathrm{E}|N|^{2(b+1)}}{(b+1)(2 b+1)} \sum_{k=0}^{\infty} \frac{(-1)^{k}}{(2 k+1)^{2(b+1)}}
\end{aligned}
$$

and for any $b>-1 / 2$,

$$
\begin{aligned}
& \lim _{\varepsilon \nearrow \infty} \varepsilon^{-2(b+1)} \sum_{n=1}^{\infty} \frac{(\log \log n)^{b}}{n^{3 / 2} \log n} \mathrm{E}\left\{\sigma \varepsilon \sqrt{\frac{\pi^{2} n}{8 \log \log n}}-M_{n}\right\}_{+} \\
= & \frac{\Gamma(b+1 / 2)}{\sqrt{2}(b+1)} \sum_{k=0}^{\infty} \frac{(-1)^{k}}{(2 k+1)^{2 b+2}},
\end{aligned}
$$

where $\Gamma(\cdot)$ is the Gamma function and $N$ stands for the standard normal random variable.

\section{Introduction}

Let $\left\{X_{n} ; n \geq 1\right\}$ be a sequence of random variables with common distribution, $\mathrm{E} X_{1}=0$ and $0<\mathrm{E} X_{1}^{2}<\infty$. Set $S_{n}=\sum_{k=1}^{n} X_{k}, M_{n}=\max _{k \leq n}\left|S_{k}\right|$, $n \geq 1$ and denote $\log x=\ln (x \vee e), \log \log x=\log (\log x)$. When $\left\{X_{n} ; n \geq 1\right\}$ is a sequence of i.i.d. random variables, Gut and Spătaru ([4]) discussed the convergence rates of the usual law of the iterated logarithm, and proved the following theorem.

Received May 11, 2008; Revised July 22, 2008

2000 Mathematics Subject Classification. 60F15, 60G50.

Key words and phrases. Chung-type law of the iterated logarithm, moment convergence rates, negative association, the law of the iterated logarithm.

Project supported by National Natural Science Foundation of China(Nos. 10671176, $10771192 \& 70871103)$. 
Theorem A. Suppose that $E X_{1}=0$ and $0<E X_{1}^{2}<\infty$. Then we have

$$
\lim _{\varepsilon \searrow 0} \varepsilon^{2} \sum_{n=1}^{\infty} \frac{1}{n \log n} P\left(\left|S_{n}\right| \geq \varepsilon \sqrt{n \log \log n}\right)=E X_{1}^{2} .
$$

Also Chow ([3]) first discussed the moment convergence of i.i.d. random variables, and got the following result.

Theorem B. Suppose that $E X=0$. Assume $p \geq 1, \alpha>1 / 2, p \alpha>1$ and $E\left(|X|^{p}+|X| \log (1+|X|)\right)<\infty$. Then for any $\varepsilon>0$,

$$
\sum_{n=1}^{\infty} n^{p \alpha-2-\alpha} E\left\{\max _{j \leq n}\left|S_{j}\right|-\varepsilon n^{\alpha}\right\}_{+}<\infty .
$$

Recently, Jiang and Zhang ([5]) obtained the precise rates in the law of the iterated logarithm for the moment convergence of i.i.d. random variables via strong approximation method. In this paper, we consider the moment convergence rates in the usual law of the iterated logarithm and the Chung-type law of the iterated logarithm for negatively associated (NA) random variables. Notice that the proof pattern is similar with that of Gut and Spătaru ([4]), and our emphasis is on the convergence rates of $M_{n}$. Also we obtained the theorems without the help of the Berry-Esseen theorem which was used in many other cases (c.f. Li [7]).

First, we shall give the definition of negatively associated random variables:

Definition 1. A finite sequence of random variables $\left\{X_{k} ; 1 \leq k \leq n\right\}$ is said to be negatively associated (NA), if for every disjoint subsets A and B of $\{1,2, \ldots, n\}$, we have

$$
\operatorname{Cov}\left\{f\left(X_{i} ; i \in A\right), g\left(X_{j} ; j \in B\right)\right\} \leq 0,
$$

whenever $f$ and $g$ are coordinatewise increasing and the covariance exists. An infinite sequence of random variables is NA if every finite subsequence is NA.

The notion of NA was first introduced by Alam and Saxena ([1]). Joag-Dev and Proschan ([6]) showed that many well known multivariate distributions possess the NA property. Because of its wide application in multivariate statistical analysis and system reliability, the notion of NA has received considerable attention recently. We refer to Joag-Dev and Proschan ([6]) for fundamental properties, Shao $([8])$ for the moment inequalities for partial sums and maximum of partial sums, and Shao and $\mathrm{Su}([9])$ for the law of the iterated logarithm, which holds under finite variance.

The content of the paper is organized as follows. In Section 2 we list the main results. The proofs of Theorems 2.1 and 2.2 are given in Sections 3 and 4 , respectively. In what follows let $M$ and $C$ etc. denote positive constants whose values possibly vary from place to place. The notation of $a_{n} \sim b_{n}$ means that $\frac{a_{n}}{b_{n}} \rightarrow 1$ as $n \rightarrow \infty$, and $[x]$ denotes the largest integer less than $x$. 


\section{Main results}

Here and in the sequel, let $\left\{X_{n} ; n \geq 1\right\}$ be a sequence of strictly stationary $\mathrm{NA}$ random variables, $\mathrm{E} X_{1}=0,0<\mathrm{E} X_{1}^{2}<\infty$, and $0<\sigma^{2}:=\mathrm{E} X_{1}^{2}+$ $2 \sum_{k=2}^{\infty} \mathrm{E} X_{1} X_{k}<\infty(0<\sigma<\infty)$ unless it is specially mentioned. Now we are in a position to present our main results.

Theorem 2.1. For any $b>-1 / 2$, if $E|X|^{2+\delta}<\infty(0<\delta \leq 1)$, then we have

$$
\begin{aligned}
& \lim _{\varepsilon \searrow 0} \varepsilon^{2 b+1} \sum_{n=1}^{\infty} \frac{(\log \log n)^{b-1 / 2}}{n^{3 / 2} \log n} E\left\{M_{n}-\sigma \varepsilon \sqrt{2 n \log \log n}\right\}_{+} \\
= & \frac{2^{-1 / 2-b} \sigma E|N|^{2(b+1)}}{(b+1)(2 b+1)} \sum_{k=0}^{\infty} \frac{(-1)^{k}}{(2 k+1)^{2(b+1)}}
\end{aligned}
$$

and

$$
\begin{aligned}
& \lim _{\varepsilon \searrow 0} \varepsilon^{2 b+1} \sum_{n=1}^{\infty} \frac{(\log \log n)^{b-1 / 2}}{n^{3 / 2} \log n} E\left\{\left|S_{n}\right|-\sigma \varepsilon \sqrt{2 n \log \log n}\right\}_{+} \\
= & \frac{2^{-1 / 2-b} \sigma}{(b+1)(2 b+1)} E|N|^{2(b+1)},
\end{aligned}
$$

where $N$ is the standard normal random variable.

Remark 2.1. The theorem holds under finite $(2+\delta)$ th moment since the central limit theorem and weak invariance principle for NA sequences are applied, while it holds under finite variance for i.i.d. case. Also here we obtain the moment convergence rates for $M_{n}$.

Theorem 2.2. For any $b>-1 / 2$, we have

$$
\begin{aligned}
& \lim _{\varepsilon \nearrow \infty} \varepsilon^{-2(b+1)} \sum_{n=1}^{\infty} \frac{(\log \log n)^{b}}{n^{3 / 2} \log n} E\left\{\sigma \varepsilon \sqrt{\frac{\pi^{2} n}{8 \log \log n}}-M_{n}\right\}_{+} \\
= & \frac{\Gamma(b+1 / 2)}{\sqrt{2}(b+1)} \sum_{k=0}^{\infty} \frac{(-1)^{k}}{(2 k+1)^{2 b+2}},
\end{aligned}
$$

where $\Gamma(\cdot)$ is the Gamma function.

Remark 2.2. It is surprising that the precise rates in the Chung-type law of the iterated logarithm holds under finite variance, since we avoid the use of the Berry-Esseen theorem.

\section{The proof of Theorem 2.1}

In this section, we shall present the proof of Theorem 2.1. First, we give some lemmas which will be used in the following proofs. 
Lemma $3.1([2])$. Let $\{W(t) ; t \geq 0\}$ be a standard Wiener process, and let $N$ be a standard normal random variable. Then for any $x>0$

$$
\begin{aligned}
P\left\{\sup _{0 \leq s \leq 1}|W(s)| \geq x\right\} & =1-\sum_{k=-\infty}^{\infty}(-1)^{k} P\{(2 k-1) x \leq N \leq(2 k+1) x\} \\
& =4 \sum_{k=0}^{\infty}(-1)^{k} P\{N \geq(2 k+1) x\} \\
& =2 \sum_{k=0}^{\infty}(-1)^{k} P\{|N| \geq(2 k+1) x\} .
\end{aligned}
$$

In particular,

$$
P\left\{\sup _{0 \leq s \leq 1} W(s) \geq x\right\} \sim 2 P(N \geq x) \sim \frac{2}{\sqrt{2 \pi} x} \exp \left(-\frac{x^{2}}{2}\right) \text { as } x \rightarrow \infty .
$$

Also, for any $x>0$,

$$
P\left(\sup _{0 \leq s \leq 1}|W(s)| \leq x\right)=\frac{4}{\pi} \sum_{k=1}^{\infty} \frac{(-1)^{k}}{2 k+1} \exp \left\{-\frac{\pi^{2}(2 k+1)^{2}}{8 x^{2}}\right\}
$$

and

$$
P\left(\sup _{0 \leq s \leq 1}|W(s)| \leq x\right) \sim \frac{4}{\pi} \exp \left(-\frac{\pi^{2}}{8 x^{2}}\right) \text { as } x \rightarrow 0 .
$$

Lemma 3.2 ([11]). Under the moment condition of Theorem 2.1, we have

$$
\frac{M_{n}}{\sigma \sqrt{n}} \rightarrow \sup _{0 \leq s \leq 1}|W(s)| \text { and } \frac{S_{n}}{\sigma \sqrt{n}} \rightarrow N \text { in distribution. }
$$

Lemma $3.3([8])$. Let $\left\{Y_{i} ; 1 \leq i \leq n\right\}$ be a sequence of $N A$ random variables with mean zero and finite variance. Denote $S_{k}=\sum_{i=1}^{k} Y_{i}, 1 \leq k \leq n, B_{n}=$ $\sum_{i=1}^{n} E Y_{i}^{2}$, then for any $z>0, y>0$,

$$
P\left(\max _{k \leq n}\left|S_{k}\right| \geq z\right) \leq 2 P\left(\max _{k \leq n}\left|Y_{k}\right| \geq y\right)+4 \exp \left\{-\frac{z^{2}}{8 B_{n}}\right\}+4\left(\frac{B_{n}}{4\left(z y+B_{n}\right)}\right)^{z /(12 y)} .
$$

Now set $b(\varepsilon)=\exp \left(\exp \left(M / \varepsilon^{2}\right)\right), M>4$ and $0<\varepsilon<1 / 4$, say. Without loss of generality, assume $\sigma=1$.

Lemma 3.4. For any $M>4$ and $b>-1 / 2$, we have

$$
\begin{array}{r}
\lim _{\varepsilon \searrow 0} \varepsilon^{2 b+1} \sum_{n \leq b(\varepsilon)} \frac{(\log \log n)^{b-1 / 2}}{n \log n} \mid n^{-1 / 2} E\left\{M_{n}-\varepsilon \sqrt{2 n \log \log n}\right\}_{+} \\
-E\left\{\sup _{0 \leq s \leq 1}|W(s)|-\varepsilon \sqrt{2 \log \log n}\right\}_{+} \mid=0
\end{array}
$$


and

$$
\begin{array}{r}
\lim _{\varepsilon \searrow 0} \varepsilon^{2 b+1} \sum_{n \leq b(\varepsilon)} \frac{(\log \log n)^{b-1 / 2}}{n \log n} \mid n^{-1 / 2} E\left\{\left|S_{n}\right|-\varepsilon \sqrt{2 n \log \log n}\right\}_{+} \\
-E\{|N|-\varepsilon \sqrt{2 \log \log n}\}_{+} \mid=0 .
\end{array}
$$

Proof. We only give the proof of (3.1), since the proof of (3.2) is similar. Note that

$$
\begin{gathered}
\varepsilon^{2 b+1} \sum_{n \leq b(\varepsilon)} \frac{(\log \log n)^{b-1 / 2}}{n \log n} \mid n^{-1 / 2} \mathrm{E}\left\{M_{n}-\varepsilon \sqrt{2 n \log \log n}\right\}_{+} \\
-\left.\mathrm{E}\left\{\sup _{0 \leq s \leq 1}|W(s)|-\varepsilon \sqrt{2 \log \log n}\right\}_{+}\right|^{\infty} \\
\leq C \varepsilon^{2 b+1} \sum_{n \leq b(\varepsilon)} \frac{(\log \log n)^{b}}{n \log n}\left(\int_{0}^{\Gamma_{n}}+\int_{\Gamma_{n}}^{\infty}\right) \mid \mathrm{P}\left(M_{n} \geq(x+\varepsilon) \sqrt{2 n \log \log n}\right) \\
-\mathrm{P}\left(\sup _{0 \leq s \leq 1}|W(s)| \geq(x+\varepsilon) \sqrt{2 \log \log n}\right) \mid d x .
\end{gathered}
$$

Set $\Gamma_{n}=(\log \log n)^{-1 / 2} \Delta_{n}^{-1 / 2}$ and

$$
\Delta_{n}=\sup _{x}\left|\mathrm{P}\left(M_{n} \geq x \sqrt{n}\right)-\mathrm{P}\left(\sup _{0 \leq s \leq 1}|W(s)| \geq x\right)\right|
$$

and from Lemma 3.2, it follows that $\Delta_{n} \rightarrow 0$ as $n \rightarrow \infty$. Thus via Toeplitz Lemma ([10]), it immediately leads to

$$
\begin{aligned}
& \varepsilon^{2 b+1} \sum_{n \leq b(\varepsilon)} \frac{(\log \log n)^{b}}{n \log n} \int_{0}^{\Gamma_{n}} \mid \mathrm{P}\left(M_{n} \geq(x+\varepsilon) \sqrt{2 n \log \log n}\right) \\
& \quad-\mathrm{P}\left(\sup _{0 \leq s \leq 1}|W(s)| \geq(x+\varepsilon) \sqrt{2 \log \log n}\right) \mid d x \\
\leq & \varepsilon^{2 b+1} \sum_{n \leq b(\varepsilon)} \frac{\Delta_{n}^{1 / 2}(\log \log n)^{b-1 / 2}}{n \log n} \\
\leq & M^{b+1 / 2} \frac{1}{(\log \log [b(\varepsilon)])^{b+1 / 2}} \sum_{n \leq b(\varepsilon)} \frac{\Delta_{n}^{1 / 2}(\log \log n)^{b-1 / 2}}{n \log n} \rightarrow 0 \quad \text { as } \varepsilon \searrow 0 .
\end{aligned}
$$


Also observe that

$$
\begin{aligned}
\varepsilon^{2 b+1} \sum_{n \leq b(\varepsilon)} \frac{(\log \log n)^{b}}{n \log n} \int_{\Gamma_{n}}^{\infty}\left(\mathrm{P}\left(M_{n} \geq(x+\varepsilon) \sqrt{2 n \log \log n}\right)\right. \\
\left.-\mathrm{P}\left(\sup _{0 \leq s \leq 1}|W(s)| \geq(x+\varepsilon) \sqrt{2 \log \log n}\right)\right) d x \\
=: \varepsilon^{2 b+1} \sum_{n \leq b(\varepsilon)} \frac{(\log \log n)^{b}}{n \log n} \int_{\Gamma_{n}}^{\infty}\left(\left(I I_{1}\right)-\left(I I_{2}\right)\right) d x .
\end{aligned}
$$

Now we deal with $\left(I I_{1}\right)$ and $\left(I I_{2}\right)$, respectively. For $\left(I I_{1}\right)$, denote $\theta=\sqrt{1 / \mathrm{E} X_{1}^{2}}$, and therefore by using Lemma 3.3 (where we take $z=(x+\varepsilon) \sqrt{2 n \log \log n}, y=$ $\beta z$ and $\left.B_{n}=n / \theta^{2}\right)$, it leads to

$$
\begin{aligned}
& \int_{\Gamma_{n}}^{\infty}\left(I I_{1}\right) d x \\
\leq & \int_{\Gamma_{n}}^{\infty}\left(2 n \mathrm{P}\left\{\left|X_{1}\right| \geq \beta(x+\varepsilon) \sqrt{2 n \log \log n}\right\}+4 \exp \left\{-\frac{\theta^{2}(x+\varepsilon)^{2} \log \log n}{4}\right\}\right. \\
& \left.+4\left(\frac{n / \theta^{2}}{8 \beta(x+\varepsilon)^{2} n \log \log n}\right)^{1 /(12 \beta)}\right) d x \\
\leq & C(\log \log n)^{-1} \int_{\Gamma_{n}}^{\infty}(x+\varepsilon)^{-2} d x \leq C(\log \log n)^{-1 / 2} \Delta_{n}{ }^{1 / 2} .
\end{aligned}
$$

As to $\left(I I_{2}\right)$, we have that, by Lemma 3.1 , for any $m \geq 1$ and $x>0$,

$$
\begin{aligned}
2 \sum_{k=0}^{2 m+1}(-1)^{k} \mathrm{P}\{|N| \geq(2 k+1) x\} & \leq \mathrm{P}\left\{\sup _{0 \leq s \leq 1}|W(s)| \geq x\right\} \\
& \leq 2 \sum_{k=0}^{2 m}(-1)^{k} \mathrm{P}\{|N| \geq(2 k+1) x\} .
\end{aligned}
$$

Thus it provides that

$$
\begin{aligned}
\int_{\Gamma_{n}}^{\infty}\left(I I_{2}\right) d x & =\int_{\Gamma_{n}}^{\infty} \mathrm{P}\left(\sup _{0 \leq s \leq 1}|W(s)| \geq(x+\varepsilon) \sqrt{2 \log \log n}\right) d x \\
& \leq 2 \sum_{k=0}^{2 m}(-1)^{k} \int_{\Gamma_{n}}^{\infty} \mathrm{P}(|N| \geq(2 k+1)(x+\varepsilon) \sqrt{2 \log \log n}) d x \\
& \leq 2 \sum_{k=0}^{2 m} \frac{(-1)^{k}}{(2 k+1)^{2}}(\log \log n)^{-1} \int_{\Gamma_{n}}^{\infty}(x+\varepsilon)^{-2} d x \\
& \leq C(\log \log n)^{-1 / 2} \Delta_{n}{ }^{1 / 2} .
\end{aligned}
$$

Then an application of Toeplitz Lemma ([10]) again, combined with (3.3), completes the proof. 
Lemma 3.5. For $0<\varepsilon<1 / 4$ and $b>-1 / 2$, we have uniformly

$$
\lim _{M \rightarrow \infty} \varepsilon^{2 b+1} \sum_{n>b(\varepsilon)} \frac{(\log \log n)^{b-1 / 2}}{n \log n} E\left\{\sup _{0 \leq s \leq 1}|W(s)|-\varepsilon \sqrt{2 \log \log n}\right\}_{+}=0 .
$$

Proof. For $k$ large enough, we obtain

$$
\begin{aligned}
& \varepsilon^{2 b+1} \sum_{n>b(\varepsilon)} \frac{(\log \log n)^{b-1 / 2}}{n \log n} \int_{0}^{\infty} \mathrm{P}\left\{\sup _{0 \leq s \leq 1}|W(s)| \geq \varepsilon \sqrt{2 \log \log n}+x\right\} d x \\
\leq & \sqrt{2} \varepsilon^{2 b+1} \sum_{n>b(\varepsilon)} \frac{(\log \log n)^{b}}{n \log n} \int_{0}^{\infty} \mathrm{P}\{N \geq(x+\varepsilon) \sqrt{2 \log \log n}\} d x \\
\leq & C \varepsilon^{2 b+1} \sum_{n>b(\varepsilon)} \frac{(\log \log n)^{b}}{n \log n} \int_{0}^{\infty} \frac{\mathrm{E}|N|^{k}}{(x+\varepsilon)^{k}(\log \log n)^{k / 2}} d x \\
\leq & C \varepsilon^{2 b+1} \sum_{n>b(\varepsilon)} \frac{(\log \log n)^{b-k / 2}}{n \log n} \varepsilon^{-k+1}=C M^{b+1-k / 2} \rightarrow 0,
\end{aligned}
$$

when $M \rightarrow \infty$, uniformly for $0<\varepsilon<1 / 4$.

Lemma 3.6. For $b>-1 / 2$, if $E\left|X_{1}\right|^{2+\delta}<\infty(0<\delta \leq 1)$, then

$$
\lim _{M \rightarrow \infty} \lim _{\varepsilon \backslash 0} \varepsilon^{2 b+1} \sum_{n>b(\varepsilon)} \frac{(\log \log n)^{b-1 / 2}}{n^{3 / 2} \log n} E\left\{M_{n}-\varepsilon \sqrt{2 n \log \log n}\right\}_{+}=0 .
$$

Proof. Write $\theta=\sqrt{1 / \mathrm{E} X_{1}^{2}}$.

By applying Lemma 3.3 (where $z=(x+\varepsilon) \sqrt{2 n \log \log n}, y=\beta^{\prime} z$ and $B_{n}=$ $\left.n / \theta^{2}\right)$ again, similarly with Lemma 3.4 , we have

$$
\begin{aligned}
& \varepsilon^{2 b+1} \sum_{n>b(\varepsilon)} \frac{(\log \log n)^{b-1 / 2}}{n^{3 / 2} \log n} \mathrm{E}\left\{M_{n}-\varepsilon \sqrt{2 n \log \log n}\right\}_{+} \\
\leq & C \varepsilon^{2 b+2} M^{-1-\delta / 2} \sum_{n>b(\varepsilon)} \frac{1}{n^{1+\delta / 2}}+C \int_{\theta^{2} M / 4}^{\infty} s^{b} e^{-s} d s+C \varepsilon^{-2} \frac{1}{\log \log b(\varepsilon)} .
\end{aligned}
$$

Then we can get the desired result immediately by letting $\varepsilon \rightarrow 0$ and $M \rightarrow$ $\infty$.

Proposition 3.1. For any $b>-1 / 2$, we have

$$
\begin{aligned}
& \lim _{\varepsilon \searrow 0} \varepsilon^{2 b+1} \sum_{n=1}^{\infty} \frac{(\log \log n)^{b-1 / 2}}{n \log n} E\left\{\sup _{0 \leq s \leq 1}|W(s)|-\varepsilon \sqrt{2 \log \log n}\right\}_{+} \\
= & \frac{2^{-1 / 2-b} E|N|^{2(b+1)}}{(b+1)(2 b+1)} \sum_{k=0}^{\infty} \frac{(-1)^{k}}{(2 k+1)^{2(b+1)}}
\end{aligned}
$$


and

$$
\lim _{\varepsilon \searrow 0} \varepsilon^{2 b+1} \sum_{n=1}^{\infty} \frac{(\log \log n)^{b-1 / 2}}{n \log n} E\{|N|-\varepsilon \sqrt{2 \log \log n}\}_{+}=\frac{2^{-1 / 2-b} E|N|^{2(b+1)}}{(b+1)(2 b+1)},
$$

where $N$ is the standard normal random variable.

Proof. Note that for any $m \geq 1$ and $x>0$, (3.4) holds. Thus it follows that for any $t>0$

$$
\begin{aligned}
\mathrm{E}\left\{\sup _{0 \leq s \leq 1}|W(s)|-t\right\}_{+} & =\int_{0}^{\infty} \mathrm{P}\left(\sup _{0 \leq s \leq 1}|W(s)| \geq t+x\right) d x \\
& \leq 2 \sum_{k=0}^{2 m}(-1)^{k} \int_{0}^{\infty} \mathrm{P}(|N| \geq(2 k+1)(t+x)) d x \\
& =2 \sum_{k=0}^{2 m} \frac{(-1)^{k}}{2 k+1} \int_{0}^{\infty} \mathrm{P}(|N| \geq(2 k+1) t+x) d x \\
& =2 \sum_{k=0}^{2 m} \frac{(-1)^{k}}{2 k+1} \mathrm{E}\{|N|-(2 k+1) t\}_{+}
\end{aligned}
$$

and

$$
\mathrm{E}\left\{\sup _{0 \leq s \leq 1}|W(s)|-t\right\}_{+} \geq 2 \sum_{k=0}^{2 m+1} \frac{(-1)^{k}}{2 k+1} \mathrm{E}\{|N|-(2 k+1) t\}_{+} .
$$

So, it suffices to show that for any $q \geq 1$ and $b>-1 / 2$,

$\lim _{\varepsilon \searrow 0} \varepsilon^{2 b+1} \sum_{n=1}^{\infty} \frac{(\log \log n)^{b-1 / 2}}{n \log n} \mathrm{E}\{|N|-q \varepsilon \sqrt{2 \log \log n}\}_{+}=q^{-2 b-1} \frac{2^{-1 / 2-b} \mathrm{E}|N|^{2(b+1)}}{(b+1)(2 b+1)}$.

Obviously,

$$
\begin{aligned}
& \lim _{\varepsilon \searrow 0} \varepsilon^{2 b+1} \sum_{n=1}^{\infty} \frac{(\log \log n)^{b-1 / 2}}{n \log n} \mathrm{E}\{|N|-q \varepsilon \sqrt{2 \log \log n}\}_{+} \\
= & \lim _{\varepsilon \searrow 0} \varepsilon^{2 b+1} \int_{e^{e}}^{\infty} \frac{(\log \log y)^{b-1 / 2}}{y \log y} \int_{q \varepsilon \sqrt{2 \log \log y}}^{\infty} \mathrm{P}\{|N| \geq x\} d x d y \\
= & 2^{1 / 2-b} q^{-2 b-1} \lim _{\varepsilon \searrow 0} \int_{\sqrt{2} q \varepsilon}^{\infty} z^{2 b} \int_{z}^{\infty} \mathrm{P}\{|N| \geq x\} d x d z \\
= & q^{-2 b-1} \frac{2^{-1 / 2-b} \mathrm{E}|N|^{2(b+1)}}{(b+1)(2 b+1)} .
\end{aligned}
$$

Thus the proposition is now proved by taking $q=2 k+1$ and $q=1$, respectively. 
Proposition 3.2. For any $b>-1 / 2$, we have

$$
\begin{aligned}
\lim _{\varepsilon \searrow 0} \varepsilon^{2 b+1} \sum_{n=1}^{\infty} \frac{(\log \log n)^{b-1 / 2}}{n \log n} \mid & n^{-1 / 2} E\left\{M_{n}-\varepsilon \sqrt{2 n \log \log n}\right\}_{+} \\
& -E\left\{\sup _{0 \leq s \leq 1}|W(s)|-\varepsilon \sqrt{\log \log n}\right\}_{+} \mid=0
\end{aligned}
$$

and

$$
\begin{array}{r}
\lim _{\varepsilon \searrow 0} \varepsilon^{2 b+1} \sum_{n=1}^{\infty} \frac{(\log \log n)^{b-1 / 2}}{n \log n} \mid n^{-1 / 2} E\left\{\left|S_{n}\right|-\varepsilon \sqrt{2 n \log \log n}\right\}_{+} \\
-E\{|N|-\varepsilon \sqrt{\log \log n}\}_{+} \mid=0 .
\end{array}
$$

Proof. It is trivial from Lemmas 3.4-3.6.

The Proof of Theorem 2.1. By using the Propositions 3.1 and 3.2, we can easily get the conclusions.

\section{The proof of Theorem 2.2}

Similarly, we state some lemmas before showing the proof of Theorem 2.2.

Lemma 4.1. For any $b>-1 / 2$ and $M>4$, we have

$$
\begin{aligned}
\lim _{\varepsilon \nearrow^{\infty}} \varepsilon^{-2(b+1)} \sum_{n \leq b(1 / \varepsilon)} \frac{(\log \log n)^{b}}{n \log n} \mid n^{-1 / 2} E\left\{\varepsilon \sqrt{\frac{\pi^{2} n}{8 \log \log n}}-M_{n}\right\}_{+} \\
-E\left\{\varepsilon \sqrt{\left.\frac{\pi^{2}}{8 \log \log n}-\sup _{0 \leq s \leq 1}|W(s)|\right\}_{+} \mid=0 .}\right.
\end{aligned}
$$

Proof. Take $\Delta_{n}=\sup _{x}\left|\mathrm{P}\left(M_{n} \leq x \sqrt{n}\right)-\mathrm{P}\left(\sup _{0<s<1}|W(s)| \leq x\right)\right|$ here, and thus via Toeplitz Lemma $([10])$, we get the desired result by following the lines in Lemma 3.4.

Lemma 4.2. For $\varepsilon>0$ sufficiently large and $b>-1 / 2$, we have

$$
\lim _{M \rightarrow \infty} \varepsilon^{-2(b+1)} \sum_{n>b(1 / \varepsilon)} \frac{(\log \log n)^{b}}{n \log n} E\left\{\varepsilon \sqrt{\frac{\pi^{2}}{8 \log \log n}}-\sup _{0 \leq s \leq 1}|W(s)|\right\}_{+}=0
$$

uniformly in $\varepsilon$. 
Proof. By virtue of Lemma 3.1, we have that

$$
\begin{aligned}
& \varepsilon^{-2(b+1)} \sum_{n>b(1 / \varepsilon)} \frac{(\log \log n)^{b}}{n \log n} \mathrm{E}\left\{\varepsilon \sqrt{\frac{\pi^{2}}{8 \log \log n}}-\sup _{0 \leq s \leq 1}|W(s)|\right\}_{+} \\
= & \varepsilon^{-2(b+1)} \sum_{n>b(1 / \varepsilon)} \frac{(\log \log n)^{b}}{n \log n} \int_{0}^{\varepsilon \sqrt{\frac{\pi^{2}}{8 \log n}}} \mathrm{P}\left\{\sup _{0 \leq s \leq 1}|W(s)| \leq t\right\} d t \\
\leq & C \int_{M}^{\infty} y^{b-1 / 2} e^{-y} d y \rightarrow 0 \text { as } M \rightarrow \infty .
\end{aligned}
$$

Lemma 4.3. There exist constants $\lambda>0$ and $C>0$ such that for any $x \geq 1$ and $n \geq 1$,

$$
P\left\{M_{n} \leq x \sqrt{n / \log \log n}\right\} \leq C \exp \left(-\frac{\lambda \log \log n}{2 x^{2}}\right) .
$$

Proof. It follows from Lemma 3.2 that $\mathrm{P}\left(S_{m} \leq 2 \sqrt{m}\right) \rightarrow \mathrm{P}(N \leq 2)$ as $m \rightarrow \infty$. Thus, there exist $\lambda>0$ and $m_{0}$ such that $\mathrm{P}\left(S_{m} \leq 2 \sqrt{m}\right) \leq e^{-\lambda}<1$, when $m \geq m_{0} / 2$. Set $m=\left[n x^{2} / \log \log n\right]$ and $N=[n /(m+1)]$. Thus, when $x \geq 1$ and $n \geq m_{0}^{2}$, we have $m \geq m_{0} / 2$. Notice that $S_{k(m+1)}-S_{(k-1)(m+1)}, k=1, \ldots, N$, are also negatively associated. Hence, for those $x \geq 1$ and $n \geq m_{0}^{2}$ satisfying $x^{2} / \log \log n<1 / 4$, we have

$$
\begin{aligned}
& \mathrm{P}\left\{M_{n} \leq x \sqrt{\frac{n}{\log \log n}}\right\} \leq \mathrm{P}\left\{M_{n} \leq \sqrt{m+1}\right\} \\
\leq & \mathrm{P}\left\{\left|S_{k(m+1)}-S_{(k-1)(m+1)}\right| \leq 2 \sqrt{m+1} ; k=1,2, \ldots, N\right\} \\
\leq & \mathrm{P}\left\{S_{k(m+1)}-S_{(k-1)(m+1)} \leq 2 \sqrt{m+1} ; k=1,2, \ldots, N\right\} \\
\leq & \prod_{k=1}^{N} \mathrm{P}\left\{S_{k(m+1)}-S_{(k-1)(m+1)} \leq 2 \sqrt{m+1}\right\} \\
= & \mathrm{P}^{N}\left\{S_{m+1} \leq 2 \sqrt{m+1}\right\} \leq e^{-\lambda N} \\
\leq & \exp \left\{-\lambda\left(\frac{n}{m+1}-1\right)\right\} \leq \exp \left\{-\lambda\left(\frac{n}{2 m}-1\right)\right\} \\
\leq & \exp \left\{-\lambda\left(\frac{\log \log n}{2 x^{2}}-1\right)\right\} \leq e^{\lambda} \exp \left\{-\frac{\lambda \log \log n}{2 x^{2}}\right\} .
\end{aligned}
$$

On the other hand, if $x^{2} / \log \log n>1 / 4$, then

$$
\mathrm{P}\left\{M_{n} \leq x \sqrt{n / \log \log n}\right\} \leq 1 \leq e^{2 \lambda} \exp \left\{-\frac{\lambda \log \log n}{2 x^{2}}\right\} .
$$

Moreover, when $x \geq 1$ and $n \leq m_{0}^{2}$, we have

$$
\mathrm{P}\left\{M_{n} \leq x \sqrt{n / \log \log n}\right\} \leq 1 \leq e^{\lambda \log \log m_{0}^{2}} \exp \left\{-\frac{\lambda \log \log n}{2 x^{2}}\right\} .
$$


Hence, the conclusion follows, as desired.

Lemma 4.4. Uniformly for $\varepsilon$ sufficiently large, we have for $b>-1 / 2$,

$$
\lim _{M \rightarrow \infty} \varepsilon^{-2(b+1)} \sum_{n>b(1 / \varepsilon)} \frac{(\log \log n)^{b}}{n^{3 / 2} \log n} E\left\{\varepsilon \sqrt{\frac{\pi^{2} n}{8 \log \log n}}-M_{n}\right\}_{+}=0 .
$$

Proof. Notice that for any $\varepsilon$ large enough, it follows from Lemma 4.3 that

$$
\begin{aligned}
& \varepsilon^{-2(b+1)} \sum_{n>b(1 / \varepsilon)} \frac{(\log \log n)^{b}}{n^{3 / 2} \log n} \mathrm{E}\left\{\varepsilon \sqrt{\left.\frac{\pi^{2} n}{8 \log \log n}-M_{n}\right\}_{+}}\right. \\
\leq & C \varepsilon^{-2 b-1} \sum_{n>b(1 / \varepsilon)} \frac{(\log \log n)^{b-1 / 2}}{n \log n} \mathrm{P}\left(M_{n} \leq \varepsilon \sqrt{\frac{\pi^{2} n}{8 \log \log n}}\right) \\
\leq & C \varepsilon^{-2 b-1} \sum_{n>b(1 / \varepsilon)} \frac{(\log \log n)^{b-1 / 2}}{n \log n} \exp \left(-\frac{4 \lambda \log \log n}{\varepsilon^{2} \pi^{2}}\right) \\
\leq & C \int_{M}^{\infty} y^{b-1 / 2} \exp \left(-\frac{4 \lambda}{\pi^{2}} y\right) d y \rightarrow 0 \text { as } M \rightarrow \infty .
\end{aligned}
$$

Proposition 4.1. For any $b>-1 / 2$, we have

$$
\begin{aligned}
& \lim _{\varepsilon \nearrow^{\infty}} \varepsilon^{-2(b+1)} \sum_{n=1}^{\infty} \frac{(\log \log n)^{b}}{n \log n} E\left\{\varepsilon \sqrt{\frac{\pi^{2}}{8 \log \log n}}-\sup _{0 \leq s \leq 1}|W(s)|\right\}_{+} \\
= & \frac{\Gamma(b+1 / 2)}{\sqrt{2}(b+1)} \sum_{k=0}^{\infty} \frac{(-1)^{k}}{(2 k+1)^{2 b+2}} .
\end{aligned}
$$

Proof. It follows from Lemma 3.1 that

$$
\begin{aligned}
& \lim _{\varepsilon \nearrow \infty} \varepsilon^{-2(b+1)} \sum_{n=1}^{\infty} \frac{(\log \log n)^{b}}{n \log n} \mathrm{E}\left\{\varepsilon \sqrt{\frac{\pi^{2}}{8 \log \log n}}-\sup _{0 \leq s \leq 1}|W(s)|\right\}_{+} \\
= & \lim _{\varepsilon \nearrow^{\prime}} \varepsilon^{-2(b+1)} \sum_{n=1}^{\infty} \frac{(\log \log n)^{b}}{n \log n} \int_{0}^{\varepsilon \sqrt{\frac{\pi^{2}}{8 \log n}}} \mathrm{P}\left\{\sup _{0 \leq s \leq 1}|W(s)| \leq t\right\} d t \\
= & \lim _{\varepsilon \nearrow^{\infty}} \varepsilon^{-2(b+1)} \sum_{n=1}^{\infty} \frac{(\log \log n)^{b}}{n \log n} \int_{0}^{\varepsilon \sqrt{\frac{\pi^{2}}{8 \log \log n}}} \frac{4}{\pi} \sum_{k=0}^{\infty} \frac{(-1)^{k}}{2 k+1} \exp \left\{-\frac{\pi^{2}(2 k+1)^{2}}{8 t^{2}}\right\} d t \\
= & \frac{4}{\pi} \sum_{k=0}^{\infty} \frac{(-1)^{k}}{2 k+1} \lim _{\varepsilon \nearrow \infty} \varepsilon^{-2(b+1)} \int_{e^{e}}^{\infty} \frac{(\log \log x)^{b}}{x \log x} \int_{0}^{\varepsilon \sqrt{\frac{\pi^{2}}{8 \log x}}} \exp \left\{-\frac{\pi^{2}(2 k+1)^{2}}{8 t^{2}}\right\} d t d x
\end{aligned}
$$




$$
\begin{aligned}
& =\frac{1}{\sqrt{2}} \sum_{k=0}^{\infty}(-1)^{k} \lim _{\varepsilon \nearrow \infty} \varepsilon^{-2(b+1)} \int_{e^{e}}^{\infty} \frac{(\log \log x)^{b}}{x \log x} \int_{(2 k+1)^{2} \log \log x / \varepsilon^{2}}^{\infty} y^{-3 / 2} e^{-y} d y d x \\
& =\frac{1}{\sqrt{2}} \sum_{k=0}^{\infty} \frac{(-1)^{k}}{(2 k+1)^{2 b+2}} \lim _{\varepsilon \nearrow \infty} \int_{(2 k+1)^{2} / \varepsilon^{2}}^{\infty} s^{b} \int_{s}^{\infty} y^{-3 / 2} e^{-y} d y d s \\
& =\frac{1}{\sqrt{2}} \sum_{k=0}^{\infty} \frac{(-1)^{k}}{(2 k+1)^{2 b+2}} \lim _{\varepsilon \nearrow \infty} \int_{(2 k+1)^{2} / \varepsilon^{2}}^{\infty} y^{-3 / 2} e^{-y} \int_{(2 k+1)^{2} / \varepsilon^{2}}^{y} s^{b} d s d y \\
& =\frac{1}{\sqrt{2}(b+1)} \sum_{k=0}^{\infty} \frac{(-1)^{k}}{(2 k+1)^{2 b+2}} \lim _{\varepsilon \nearrow \infty}^{\infty} \int_{(2 k+1)^{2} / \varepsilon^{2}}^{\infty} y^{b-1 / 2} e^{-y} d y \\
& =\frac{\Gamma(b+1 / 2)}{\sqrt{2}(b+1)} \sum_{k=0}^{\infty} \frac{(-1)^{k}}{(2 k+1)^{2 b+2}} .
\end{aligned}
$$

Proposition 4.2. For any $b>-1 / 2$, we have

$$
\begin{aligned}
\limsup _{\varepsilon \nearrow \infty} \varepsilon^{-2(b+1)} \sum_{n=1}^{\infty} \frac{(\log \log n)^{b}}{n \log n} \mid & n^{-1 / 2} E\left\{\varepsilon \sigma \sqrt{\frac{n \pi^{2}}{8 \log \log n}}-M_{n}\right\}_{+} \\
& -E\left\{\varepsilon \sqrt{\left.\frac{\pi^{2}}{8 \log \log n}-\sup _{0 \leq s \leq 1}|W(s)|\right\}_{+} \mid=0 .}\right.
\end{aligned}
$$

Proof. From Lemmas 4.1, 4.2 and 4.4, it follows easily.

The Proof of Theorem 2.2. By virtue of Propositions 4.1 and 4.2, we complete the proof immediately.

Acknowledgements. The authors thank the referee for several comments that have led to improvements in this work.

\section{References}

[1] K. Alam and K. M. L. Saxena, Positive dependence in multivariate distributions, Comm. Statist. A-Theory Methods 10 (1981), no. 12, 1183-1196.

[2] P. Billingsley, Convergence of Probability Measures, John Wiley \& Sons, Inc., New YorkLondon-Sydney, 1968.

[3] Y. S. Chow, On the rate of moment convergence of sample sums and extremes, Bull. Inst. Math. Acad. Sinica 16 (1988), no. 3, 177-201.

[4] A. Gut and A. Spătaru, Precise asymptotics in the law of the iterated logarithm, Ann. Probab. 28 (2000), no. 4, 1870-1883.

[5] Y. Jiang and L. X. Zhang, Precise rates in the law of iterated logarithm for the moment of i.i.d. random variables, Acta Math. Sin. (Engl. Ser.) 22 (2006), no. 3, 781-792.

[6] K. Joag-Dev and F. Proschan, Negative association of random variables, with applications, Ann. Statist. 11 (1983), no. 1, 286-295.

[7] Y. X. Li, Precise asymptotics in complete moment convergence of moving-average processes, Statist. Probab. Lett. 76 (2006), no. 13, 1305-1315. 
[8] Q. M. Shao, A comparison theorem on moment inequalities between negatively associated and independent random variables, J. Theoret. Probab. 13 (2000), no. 2, 343-356.

[9] Q. M. Shao and C. Su, The law of the iterated logarithm for negatively associated random variables, Stochastic Process. Appl. 83 (1999), no. 1, 139-148.

[10] W. F. Stout, Almost Sure Convergence, Academic, New-York, 1995.

[11] C. Su, L. C. Zhao, and Y. B. Wang, The moment inequalities and weak convergence for negatively associated sequences, Sci. China Ser. A 40 (1997), no. 2, 172-182.

Ke-Ang Fu

College of Statistics and Mathematics

ZheJiang Gongshang University

Hangzhou 310018, P. R. China

E-mail address: statzju@tom.com

Li-HuA Hu

College of Statistics and Mathematics

ZheJiang Gongshang University

Hangzhou 310018, P. R. China

E-mail address: lihuahulihua@126.com 